\title{
Air below the diaphragm
}

\section{Hester Beckmann-Stam ${ }^{1}$ and Jan Pompe ${ }^{2}$}

${ }^{1}$ Department of Anesthesiology and Intensive Care, Bravis Hospital, the Netherlands

${ }^{2}$ Department of Intensive Care, Radboud University Hospital, the Netherlands

\section{Summary}

A 69-year-old man with a history of alcohol and nicotine abuse was intubated on our ICU because of respiratory insufficiency, two weeks after an extended left sided, blunt thoracic trauma. A chest X-ray showed the falciform ligament as a sign of massive free intraperitoneal gas. On operation there was a perforation of the small intestine, which was successfully resected and a primary anastomosis was made.

\section{Case report}

A 69-year-old man with a history of alcohol and nicotine abuse was admitted to the ICU after suffering extended left sided, blunt thoracic trauma. Trauma screening revealed a haemato-pneumothorax with pulmonary contusions, a stable fracture of the second thoracic vertebra, facial fractures with a small subdural and subarachnoid bleeding and intracerebral contusions. Furthermore he had fractures of his left scapula, clavicle and forearm. He was intubated after chest drainage, and external fixation of his forearm took place. Post-operative he developed a delirium and an ileus.

After 2 weeks he was reintubated because of respiratory insufficiency, assumedly due to a (aspiration) pneumonia. On examination we noted a body temperature of $39.1^{\circ} \mathrm{C}$, a distended abdomen, hypoactive bowel sounds, and diffuse tenderness without rebound pain. The laboratory evaluation revealed a white-cell count of $8,6 \times 109 / 1$. Antibiotics were started. Thoracic X-ray after intubation showed a possible infiltrate on the left thoracic side and subdiaphragmal air, which appeared to be free intraperitoneal air. A CT-scan confirmed this pneumoperitoneum (Figure 1).

Free air below the diaphragm visible at plain abdominal films or $\mathrm{X}$-ray is an emergency sign. Therefore it is extremely important to differentiate where the free air is located. Differentiation between free intraperitoneal air or intraluminal/intragastric air (e.g. after intubation) in this case is the clear visualization of the falciform ligament and the

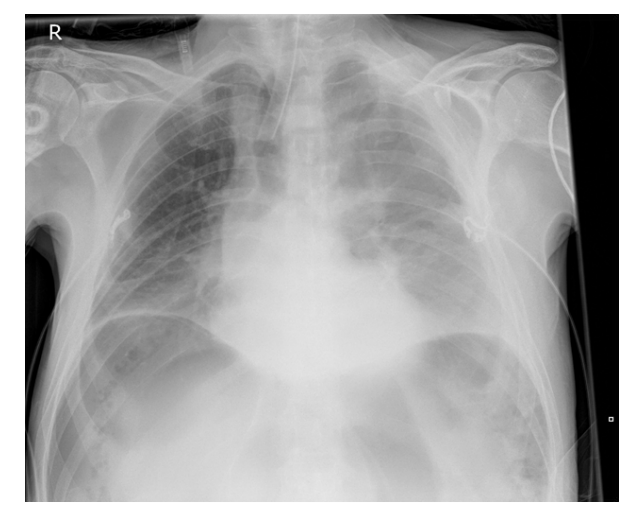

Figure 1. Chest X-ray 'air below the diaphragm'.

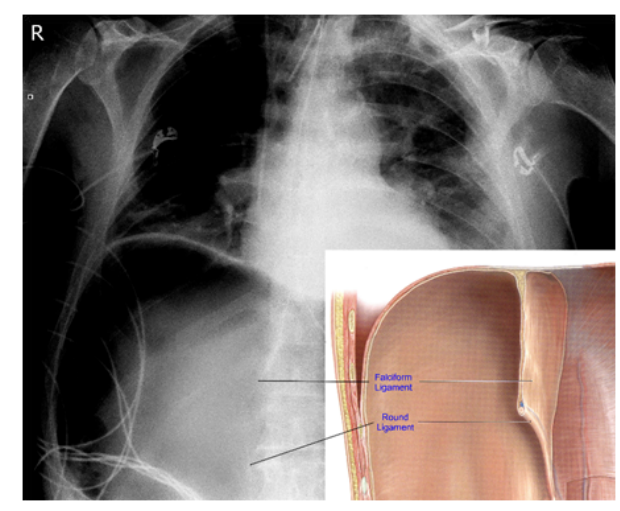

Figure 2. Chest X-ray showing falciform ligament and round ligament sign .

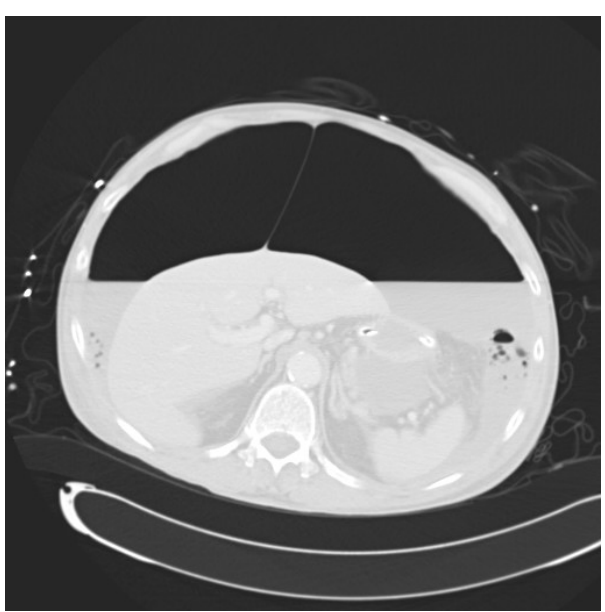

Figure 3. CT scan showing falciform ligament sign.

Correspondence to: Hester Beckmann-Stam, Department of Anesthesiology and Intensive Care, Bravis Hospital, the Netherlands, E-mail: hesterstam@yahoo.com

Received: November 19, 2016; Accepted: November 24, 2016; Published: November 26, 2016 
round ligament. The ligamentum falciforme is the broad, thin layer of folded peritoneum, attached to the upper border of the abdominal cavity and the liver. The round ligament exists in the free edge of the falciform ligament, dividing the left part of the liver, connecting the falciform ligament with the umbilicus as a remnant of the umbilical vein. These two ligaments become visible as free air accumulation develops in the right subphrenic area and ventral surface of the liver (Figures 2 and 3).
The patient was immediately taken to the operating room. On surgical exploration there was a perforation of the small intestine, which was successfully resected and a primary anastomosis was made.

The patient recovered from his surgery after a prolonged ICU stay, despite the subsequent sepsis with renal insufficiency and delirium.

Copyright: (C2016 Beckmann-Stam H. This is an open-access article distributed under the terms of the Creative Commons Attribution License, which permits unrestricted use, distribution, and reproduction in any medium, provided the original author and source are credited. 